\title{
The role of direct oral anticoagulants (DOACs) in the treatment of heparin-induced thrombocytopenia (HIT): An evidence-based literature review
}

\author{
Bright Huo', Marcel Surette', Aaron Kelly' \\ I. Class of 2019, Faculty of Pharmacy, Dalhousie University, Halifax, Nova Scotia, Canada.
}

\begin{abstract}
Heparin-induced thrombocytopenia (HIT) poses a risk of death secondary to thrombotic complications. Treatment options are limited for patients with poor IV access, as contemporary options are restricted to parenteral agents before switching to oral vitamin $\mathrm{k}$ antagonists. A literature review was conducted to examine the effectiveness of direct oral anticoagulants (DOACs) in the primary treatment of HIT. High quality evidence is scarce surrounding the use of DOACs for this indication, while past reviews have not critically appraised the evidence. Additionally, the most recent study from 2017 investigating the use of DOACs for this indication has not been reported in past literature reviews. The Cochrane Library, Embase, PubMed, Google Scholar and ClinicalTrials.gov were searched to identify and critically appraise the best available evidence. Salient literature demonstrates that DOACs are effective at raising platelet count to baseline after seven days, on average. Thrombosis and major bleeding are rarely observed when DOACs are used as primary therapy. While large scale studies are needed, patients with HIT that have poor IV access may benefit from the ease of administration, rapid onset of action and lack of routine monitoring associated with DOAC therapy.
\end{abstract}

\section{Introduction}

Used for deep vein thrombosis (DVT) prophylaxis, unfractionated (UFH) and low-molecular weight heparins (LMWH) may cause heparin-induced thrombocytopenia (HIT), an adverse drug reaction. ${ }^{1}$ HIT is immune-mediated, occurring when heparin-induced antibodies interact with platelet factor 4 (PF4). ${ }^{1}$ Activated platelets mediate the release of prothrombotic microparticles and induce platelet consumption, resulting in the development of thrombosis and eventually, thrombocytopenia. ${ }^{2}$ Defined as a 30-50\% decrease in platelet count from baseline, thrombocytopenia ensues five to ten days after exposure. ${ }^{1}$ Reduction in platelet count $>50 \%$ from baseline, a nadir of $\geq 20 \times 10^{9} / \mathrm{L}$ and skin necrosis at the heparin injection site increase the likelihood of HIT according to the 4Ts scoring tool. ${ }^{1}$ Complications of HIT include limb amputations as well as DVT and pulmonary embolism (PE). ${ }^{1}$ HIT is associated with a $5-10 \%$ mortality rate secondary to thrombotic complications. ${ }^{1}$

Treatment options for HIT include fondaparinux (Arixtra), lepirudin (Refludan), bivalirudin (Angiomax), danaparoid (Orgaran) and argatroban (Argatroban); the latter two are approved by Health Canada for this indication. ${ }^{1}$ Fondaparinux's off-label use stems from observational data and is associated with a thrombosis rate of $16 \% .^{3}$ Lepirudin is currently not available in Canada and is given IV, similarly to bivalirudin. ${ }^{1}$ There is a $25 \%$ thrombotic event rate when treated with danaparoid or argatroban. ${ }^{3}$ These parenteral anticoagulants must be transitioned to vitamin $\mathrm{K}$ antagonists (VKAs) following platelet recovery, increasing cost and risk for warfarin-induced microthrombosis. ${ }^{4}$ The high rate of thrombotic events, difficulty of administration and the need for routine monitoring with warfarin (Coumadin) necessitate alternative treatment options. ${ }^{4}$

Anecdotal reports suggest DOACs are effective in treating HIT after treatment failure with conventional agents. ${ }^{5}$ DOACs do not interact with PF4, lending them an advantage over danaparoid and fondaparinux, which may cross-react with HIT antibodies. ${ }^{1,7,8}$ Oral administration, rapid onset of action, ease of use and the lack of routine monitoring make DOACs an attractive treatment option for HIT including patients with poor IV access. ${ }^{8}$

\section{Clinical Question}

In a patient with poor IV access, would DOACs be a safe and effective option for primary treatment of HIT to reduce the risk of thrombosis and bleeding?

\section{Search Strategy}

The Cochrane Library, Embase, PubMed and Google Scholar were searched between November 12th, 2017 to February 13th, 2018. Keywords included "thrombocytopenia", "heparin-induced thrombocytopenia", "HIT", "anticoagulants", "novel oral anticoagulants", "NOACs", "direct oral anticoagulants", "DOACs", "rivaroxaban", "dabigatran", "apixaban", and "edoxaban" (Figure 1). This generated 5214 articles, which were limited by publication type (meta-analyses, systematic and literature reviews, randomized controlled trials, clinical practice guidelines, and journal articles), year 


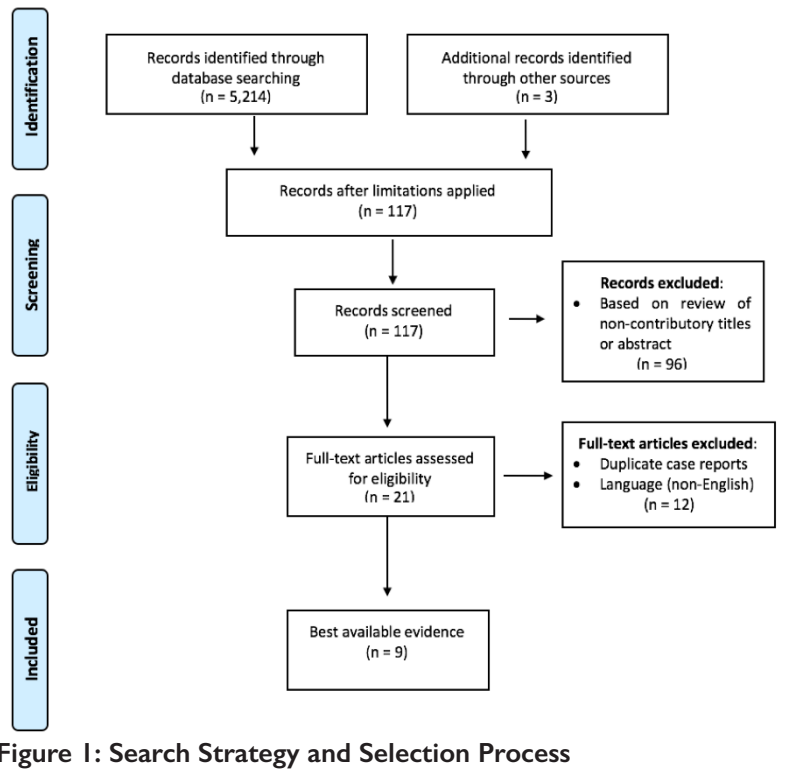

of publication (last 10 years), and language (English). Articles were screened by reviewing titles and abstracts to ensure they examined the treatment of HIT with DOACs. As such, patients switching from parenteral HIT therapy, those with comorbidities such as cancer or end-stage renal disease and specific patient populations dissimilar to the population for our clinical question such as pregnancy, nephropathy and post cardiac surgery, were excluded to mitigate potential contributors to negative confounding bias. A total of nine articles were relevant to our clinical question. The data reported in these studies are summarized in two articles, which were deemed to be the best available evidence. This included a combined retrospective cohort study ${ }^{4}$ and systematic review ${ }^{4,11}$ as well as a separate retrospective cohort study. ${ }^{9}$ ClinicalTrials.gov was searched on May 2nd, 2018 to identify studies that are ongoing, unpublished or withdrawn and yielded no additional articles.

\section{Results}

DOACs have been shown to be effective for patients with HIT, although few trials evaluate their use as treatment for this indication. Of these studies, most investigate the use of DOACs as secondary treatment for HIT following the use of conventional agents; however, studies that examine the use of DOACs as primary treatment for HIT have demonstrated positive results. The best available evidence is summarized in a combined retrospective cohort study ${ }^{4}$ and systematic review, ${ }^{4,12}$ while the most recent evidence stems from a cohort study conducted in 2017. ${ }^{9}$

A retrospective cohort study identified 16 patients in four hospitals in Hamilton, Ontario with a 4Ts score $>4$ points and a positive test for HIT antibodies. ${ }^{4}$ Patients were stratified by initial treatment with a DOAC or with a non-DOAC before bridging to a DOAC. Only 8 patients received a DOAC as primary treatment. Doses varied from 15-30mg of rivaroxaban daily, with a median treatment duration of 3 months. Patients were evaluated after 30 days to assess for thromboembolism, major bleeding and time to platelet recovery. Seven of the eight patients receiving rivaroxaban as primary treatment were thrombocytopenic before initiation of treatment, with a median platelet count of $56,000 / \mu \mathrm{L}$. No thrombotic events or major bleeding were observed. The average time to platelet recovery was 7.3 days. ${ }^{4}$

The systematic review assessed patients with a 4Ts score $\geq 4$ with HIT antibody detection.,12 Patients were included with a 4Ts score of $\geq 6$ if antibody testing was unreported. The 30-day thrombotic event rate and major bleeding rate were evaluated. While doses varied, 69 patients received rivaroxaban, dabigatran or apixaban. Rivaroxaban was assigned to 46 patients with a median platelet count of $73,000 / \mu \mathrm{L}$; of these patients, only 25 received primary treatment with a DOAC. The frequency of new, progressive or recurrent thrombosis was $2.2 \%$ (95\% CI, $0.4 \%-11.3 \%$ ). One episode of thrombosis was observed, resolving upon removal of a central venous catheter and continued treatment with rivaroxaban. No major bleeding was observed. Apixaban was assigned to 12 patients, while 11 patients received dabigatran, for a total of 23 patients with a median platelet count of $58,000 / \mu \mathrm{L}$. One patient had a thrombotic event, while none experienced major bleeding., ${ }^{4,12}$ Since this combined paper was published, new research has been conducted.

A retrospective cohort study conducted in 2017 assessed 12 patients with 4 Ts scores $\geq 4$ that tested positive for HIT antibodies. ${ }^{9}$ If serotonin release assays yielded a negative test result, patients were excluded from analysis. Primary outcomes included thromboembolism, gangrene or amputation due to critical limb ischemia during hospitalization. Nine patients received apixaban while three patients received rivaroxaban for an average of 9.33 days (ranging from one to 32 days) at varying doses. Eleven patients continued DOAC therapy post-discharge. At baseline, five patients had HIT-associated thrombosis. Of the 12 patients, seven were given argatroban before receiving a DOAC irrespective of thrombosis status. No patients experienced thrombosis or major bleeding and the mean time to platelet recovery was 7.42 days. $^{9}$

\section{Discussion}

High quality evidence supporting DOACs in the treatment of HIT is scarce. Nevertheless, the retrospective cohort study from Hamilton, Ontario showed that the use of rivaroxaban as primary treatment 
for HIT is promising. ${ }^{4}$ Explicit criteria for the diagnosis of HIT and platelet count recovery improved internal validity. Patients were evaluated after three months, allowing sufficient time to determine the efficacy and short-term safety of DOACs. However, internal validity was compromised as fondaparinux may also cause HIT $^{1}$, but the causative agent of HIT was never stated. ${ }^{4}$ Only five patients had a high probability of HIT while two were classified as intermediate probability. ${ }^{4}$ If HIT was not present and an incorrect diagnosis was reached, efficacy may have been overestimated. Heterogeneity between patient characteristics, varying doses and unreported methods of thrombosis measurement further mitigated the legitimacy of the results. As patients ranged from 54-94 years of age, older patients may have been more sensitive to adverse effects such as bleeding. Initial indications for heparin treatment varied. External validity was reduced by the small, specific subgroup of patients in Hamilton, Canada and by the variation in initial indications for heparin treatment.

The systematic review demonstrated encouraging results for the usage of rivaroxaban, dabigatran and apixaban for this indication. Search strategy, inclusion and exclusion criteria and assessment of outcomes were stated a priori, ameliorating the quality of the study design. Both English and non-English articles were searched, limiting publication bias, while a clinical diagnosis of HIT was explicitly defined. However, in patients without HIT antibody confirmation, it was not explained why other causes of thrombocytopenia were unlikely. Potential inclusion of cases of thrombocytopenia unassociated with heparin limits internal validity, as these patients may have had more favourable outcomes. ${ }^{4,12}$

Studies included in the systematic review did not adequately report patient characteristics including medical history and concurrent drug therapy. Sufficient details surrounding methods and the assessment of outcomes in these studies were limited. It was not stated how studies were assessed for quality, minimizing internal validity of the pooled results as patients may have been heterogeneous, while doses and duration of therapy were not standardized. Of the 46 patients treated with rivaroxaban, 21 patients received a parenteral anticoagulant beforehand, making it more difficult to extrapolate the results. Physicians may also have been hesitant to prescribe DOACs as long-term risks for patients under this indication have yet to be established. Thus, channeling may have been present, as patients prescribed DOACs may have had a more favorable prognosis, increasing the likelihood of achieving positive results.

Data from the most recent study published in 2017 affirms the positive results from the existing evidence, as platelets were observed to recover after a median 7.42 days with no major bleeding or thrombosis. Strengths of the study include that diagnosis of HIT, major bleeding, time to platelet recovery, inclusion criteria and outcomes were explicitly defined. The usage of serotonin release assay (SRA) boosts the quality of the study, as SRA is virtually diagnostic for HIT. ${ }^{10}$ However, SRA testing was not performed on each patient, increasing the risk of selection bias, as patients may have had more favorable outcomes if they were actually SRA negative. Short-term follow-up, inclusion of patients with HIT-related thrombosis, lack of individualized patient results, use of DOACs as secondary treatment, varying doses and small sample size minimize internal and external validity. Patient comorbidities were not reported while long-term outcomes were not assessed.

\section{Conclusion}

There is a lack of high-quality evidence investigating the use of DOACs in the treatment of HIT. There are currently no published meta-analyses or randomized controlled trials addressing this topic, while the only systematic review is small. Although scarce, evidence supporting the use of DOACs for the primary treatment of HIT is promising; especially with rivaroxaban. With demonstrable efficacy with respect to recovery of platelet count, DOACs have the potential to become viable treatment options for HIT. In comparison to standard options for HIT treatment, DOACs were reported to have a lower incidence rate of thrombotic events. $^{4,9}$

The best available evidence maintained the reputable safety profile of DOACs as there was no bleeding reported when used to treat HIT. ${ }^{4,9,12}$ Despite their relatively high cost, medication adherence is facilitated by their ease of administration, lack of monitoring requirements and possibility of oral dosing. Earlier hospital discharge is possible as they can be used post-discharge, reducing the risk and expense of switching to VKAs. ${ }^{4}$

For patients with poor IV access, treatment options for HIT are limited. Fondaparinux has been used more frequently but has a high thrombotic rate, may interact with PF4 and is more difficult to administer. ${ }^{1}$ Evidence supporting the use of fondaparinux in the treatment of HIT is weak. ${ }^{4}$ Of the DOACs, rivaroxaban has the most research supporting its use to treat HIT at a dose of $15 \mathrm{mg}$ twice daily for four weeks. ${ }^{4}$ Studies evaluating the use of edoxaban for this indication have not been conducted. Further advancements should target randomized controlled trials with large sample sizes, stratifying patients to each of the DOACs as well as 
standard treatment options with long-term follow-up. Comparative safety and efficacy data will cement the role of DOACs in the treatment of HIT.

\section{Acknowledgements}

We would like to acknowledge Emma Murray for her efforts in helping us with this literature review.

\section{Conflict of Interest Statement}

There are no conflicts of interest by any author pertaining to this submission.

\section{References}

1. Linkins L, Dans AL, Moores LK, Bona R, Davidson BL, Schulman S, et al. Treatment and Prevention of Heparin-Induced Thrombocytopenia: Antithrombotic Therapy and Prevention of Thrombosis, 9th ed: American College of Chest Physicians Evidence-Based Clinical Practice Guidelines. Chest. 2012;141(2, Supplement):e495S-e530S.

2. Zinkovsky D, Antonopoulos M. Heparin-Induced Thrombocytopenia Overview and Treatment. Phar Ther. 2008 Nov;33(11):642.

3. Kang M, Alahmadi M, Sawh S, Kovacs MJ, Lazo-Langner A.
Fondaparinux for the treatment of suspected heparin-induced thrombocytopenia: a propensity score-matched study. Blood. 2015;125(6):924-929.

4. Warkentin TE, Pai M, Linkins L. Direct oral anticoagulants for treatment of HIT: update of Hamilton experience and literature review. Blood. 2017;130(9):1104-1113.

5. Greinacher A, Selleng K, Warkentin TE. Autoimmune heparin-induced thrombocytopenia. J Thromb Haemost. 2017 Nov;15(11):2099-2114.

6. Sanidas EA, Viniou NA, Diamantopoulos P, Barbetseas J Heparin induced thrombocytopenia Contemporary therapeutic approaches in light of the new oral anticoagulants. Hamostaseologie. 2015;35(4):372-375.

7. Ho PJ, Siordia JA. Dabigatran approaching the realm of heparin-induced thrombocytopenia. Blood Res. 2016;51(2):77-87.

8. Dhakal P, Pathak R, Giri S, Murthy G, Bhatt V. New Oral Anticoagulants for the Management of Heparin Induced Thrombocytopenia: A Focused Literature Review. Cardiovasc Hematol Agents Med Chem. 2015;13:87-91.

9. Davis KA, Davis DO. Direct acting oral anticoagulants for the treatment of suspected heparin-induced thrombocytopenia. Eur J Haematol. 2017;99:332-335.

10. Warkentin, T. E., Arnold, D. M., Nazi, I. and Kelton, J. G. The platelet serotonin-release assay. Am. J. Hematol. 2015;90:564572.

11. Linkins LA et al. Rivaroxaban for the treatment of suspected or confirmed HIT study. J Thromb Haemost. 2016 Jun;14(6):120610 